\title{
DL-3-n-butylphthalide protects H9c2 cardiomyoblasts from ischemia/reperfusion injury by regulating HSP70 expression via PI3K/AKT pathway activation
}

\author{
YUNCHEN YU ${ }^{1-3 *}$, YUYING ZHU ${ }^{3 *}$, XIAOTONG SUN ${ }^{3}$, YONGXING LI $^{1}$, MINGLING WANG $^{3}$, \\ BIN DONG ${ }^{3}$, XIAODONG SUN ${ }^{4}$ and WENMING HOU ${ }^{1}$
}

\author{
${ }^{1}$ Department of Cardiovascular Surgery, Affiliated Hospital of Weifang Medical University, Weifang, Shandong 261000; \\ ${ }^{2}$ Department of Anesthesiology, Qingdao Fuwai Cardiovascular Hospital, Qingdao, Shandong 266000; \\ ${ }^{3}$ School of Anesthesiology, Shandong Provincial Medicine and Health Key Laboratory of Clinical Anesthesia, \\ Weifang Medical University; ${ }^{4}$ Department of Endocrinology, Affiliated Hospital of \\ Weifang Medical University, Weifang, Shandong 261000, P.R. China
}

Received May 20, 2021; Accepted June 21, 2021

DOI: $10.3892 / \mathrm{etm} .2021 .10441$

\begin{abstract}
DL-3-n-butylphthalide (NBP) is commonly used to treat ischemic strokes due to its antioxidative and anti-inflammatory effects. The present study aimed to examine the protective effects of NBP on myocardial ischemia-reperfusion injury (MIRI) by establishing a MIRI model in H9c2 cells. Cell viability assay using Cell Counting Kit-8, lactate dehydrogenase (LDH) cytotoxicity and lipid peroxidation malondialdehyde (MDA) content were assessed to detect cell activity, degree of cell injury and oxidative stress reaction. Reverse transcription-quantitative PCR was used to quantify the expression of inflammatory factors in H9c2 cells. Western blotting and immunofluorescence staining were used to detect the protein expression of PI3K/AKT and heat shock protein 70 (HSP70). The present results indicated that NBP significantly increased cell viability during ischemia-reperfusion. Moreover, NBP inhibited the release of LDH and the production of MDA. NBP treatment also significantly decreased the expression of inflammatory factors at the mRNA level. Additionally, NBP activated the PI3K/AKT pathway and upregulated the expression of HSP70 compared with cells in
\end{abstract}

Correspondence to: Professor Wenming Hou, Department of Cardiovascular Surgery, Affiliated Hospital of Weifang Medical University, 2428 Yuhe Road, Weifang, Shandong 261000, P.R. China E-mail: fyhouwm@wfmc.edu.cn

Professor Xiaodong Sun, Department of Endocrinology, Affiliated Hospital of Weifang Medical University, 2428 Yuhe Road, Weifang, Shandong 261000, P.R. China

E-mail: xiaodong.sun@wfmc.edu.cn

${ }^{*}$ Contributed equally

Key words: dl-3-n-butylphthalide, myocardial ischemia-reperfusion injury, PI3K/AKT, heat shock protein 70, H9c2 cells the MIRI model. LY294002, a PI3K inhibitor, reversed the protective effects of NBP and suppressed the expression of HSP70. The present study demonstrated that NBP protected H9c2 cells from MIRI by regulating HSP70 expression via $\mathrm{PI} 3 \mathrm{~K} / \mathrm{AKT}$ pathway activation.

\section{Introduction}

With the wide application of intervention and cardiac surgery in heart disease, myocardial ischemia-reperfusion injury (MIRI) has increased, becoming an urgent problem in the medical field. The main consequence of ischemia is bioenergetic exhaustion caused by an insufficient transport of oxygen and nutrients (1). MIRI is a phenomenon in which the ischemic injury of tissues and organs is not alleviated, but further aggravated after blood perfusion; it is caused by an increase in oxygen free radical content, calcium overload, inflammatory cell infiltration and other phenomena associated with ischemia-reperfusion, leading to cardiomyocyte necrosis and apoptosis (2). However, timely reperfusion is effective to resuscitate myocardial tissue and improve clinical prognosis, paradoxically, this type of reperfusion therapy causes more severe myocardial injury than simple ischemia (3). A previous study has reported that certain interventions, such as primary unloading, could limit infarct size after acute myocardial infarction (4). In addition, a number of anti-ischemia-reperfusion drugs have emerged, but their effect in clinical application has not been exceptional (5). Therefore, the mechanism of reperfusion injury needs to be further elucidated.

Heat shock proteins (HSPs) are highly conserved proteins. The expression of HSPs is induced to protect body functions during stress exposure (6). HSP70 is an endogenous protective protein that plays a protective role in MIRI $(7,8)$. The PI3K/AKT signaling pathway has also been indicated to serve an important role in the protection of cardiomyocytes in MIRI (9-11). The PI3K family is divided into class I, II and III forms, class I being more commonly detected in MIRI (12). Previous studies have indicated that apoptosis 
during reperfusion is an important cause of fatal cardiomyocyte injury. The remedial kinase pathway against apoptosis during reperfusion is called the reperfusion injury salvage kinase (RISK) pathway (13). The PI3K/AKT pathway is one of the RISK signaling pathways (14), which functions through the regulation of cell morphology, cardiomyocyte survival, apoptosis, protein synthesis and metabolic integration (15). Previous studies have identified a close association between the PI3K/AKT pathway and HSP70, and this mutual regulation has been demonstrated in brain and lung tissues $(16,17)$. However, the association between PI3K/AKT and HSP70 in the myocardium requires further elucidation.

DL-3-n-butylphthalide (NBP) is a natural product extracted from celery seeds (18). Previous studies have revealed that NBP promoted vasodilation and improved cerebral microcirculation by promoting the production of nitric oxide $(19,20)$. Furthermore, NBP has been indicated to stabilize the blood-brain barrier and mitochondrial membrane structure by inhibiting inflammation and oxidative stress (21-24). Interestingly, NBP has also been hypothesized to play an important role in MIRI by reducing myocardial infarction and the incidence of arrhythmia $(25,26)$. Recent studies have indicated that NBP significantly improved cardiac function by regulating the PI3K/AKT pathway in MIRI, and that HSP70 exhibited protective effects on MIRI $(7,27)$. However, the role of HSP70 in the cardioprotective effect of NBP remains unknown. Furthermore, further research is required to explore the relationship between HSP70 and the PI3K/AKT signaling pathway.

Therefore, the present study aimed to explore whether NBP protected against MIRI by reducing inflammation and oxidative stress through the PI3K/AKT signaling pathway and HSP70. The association between NBP, PI3K/AKT and HSP70 was also explored.

\section{Materials and methods}

Materials and reagents. NBP (cat. no. H20100041) was purchased from CSPC Enbipu Pharmaceutical Co., Ltd. LY294002 (cat. no. A8250; PI3K inhibitor) was purchased from APeXBIO Technology LLC. FBS (cat. no. SFBS) was purchased from Bovogen Biologicals Pty Ltd. DMEM (low-glucose, cat. no. 30021; high-glucose, cat. no. 30022) was purchased from HyClone; Cytiva. ChamQ Universal SYBR qPCR Master Mix (cat. no. Q711-02) and HiScript II Q RT SuperMix for qPCR + gDNA wiper (cat. no. R223-01) were purchased from Vazyme Biotech Co., Ltd. All antibodies and reagents were of analytical grade and are commercially available.

Cell culture. H9c2 cells (cat. no. CL-0089) were obtained from Procell Life Science \& Technology Co., Ltd. H9c2 cells $\left(1 \times 10^{6}\right.$ cells $\left./ \mathrm{ml}\right)$ were seeded in $25-\mathrm{cm}^{2}$ cell-culture flasks containing high-glucose DMEM (10\% FBS; $1 \%$ penicillin/streptomycin). The cells were cultured in a cell incubator with $95 \%$ air and $5 \% \mathrm{CO}_{2}$ at $37^{\circ} \mathrm{C}$. After $\mathrm{H} 9 \mathrm{c} 2$ cells were cultured for $24 \mathrm{~h}$, they were randomly assigned to four groups: i) Control group (CON); ii) MIRI group; iii) NBP pretreatment group (MIRI + NBP); and iv) PI3K inhibitor group (MIRI + NBP + LY294002). CON cells were cultured at $37^{\circ} \mathrm{C}$ with $95 \%$ air. MIRI cells were incubated at $37^{\circ} \mathrm{C}$ with $5 \% \mathrm{CO}_{2}, 93 \% \mathrm{~N}_{2}$ and $2 \% \mathrm{O}_{2}$ for $6 \mathrm{~h}$, then reoxygenated for $4 \mathrm{~h}$. MIRI + NBP cells were pretreated with $100 \mu \mathrm{M} \mathrm{NBP}$ for $2 \mathrm{~h}$ before being subjected to hypoxia for $6 \mathrm{~h}$, followed by reoxygenation for $4 \mathrm{~h}$. MIRI + NBP + LY294002 cells were pretreated with $10 \mu \mathrm{M}$ LY294002 (28) for $1 \mathrm{~h}$ before treatment with NBP for $2 \mathrm{~h}$, followed by hypoxia for $6 \mathrm{~h}$ and reoxygenation for $4 \mathrm{~h}$.

Construction of the H9c2 MIRI model. To simulate the in vivo model of ischemia-reperfusion injury, the cell culture medium was replaced with low-glucose DMEM without FBS, and the cells were incubated at $37^{\circ} \mathrm{C}$ with $5 \% \mathrm{CO}_{2}, 93 \% \mathrm{~N}_{2}$ and $2 \% \mathrm{O}_{2}$. A total of $10 \mu \mathrm{M}$ of LY294002 was added $1 \mathrm{~h}$ before NBP treatment. NBP pretreatment lasted for $2 \mathrm{~h}$ before hypoxia. After MIRI, the medium was replaced with high-glucose DMEM (10\% FBS; $1 \%$ penicillin/streptomycin) and the cells were cultured in an incubator $\left(37^{\circ} \mathrm{C} ; 5 \% \mathrm{CO}_{2} ; 95 \%\right.$ air $)$ for $4 \mathrm{~h}$.

Determination of optimal NBP concentration and cell viability. $\mathrm{H} 9 \mathrm{c} 2$ cells $\left(1 \times 10^{4}\right.$ cells/well) were seeded in 96-well plates for 24-48 h, and then pretreated with different doses of $\operatorname{NBP}(1,50,100,200,300$ and $500 \mu \mathrm{M})$. After reperfusion, the original medium was discarded and $100 \mu \mathrm{l}$ medium containing 10 mg/ml Cell Counting Kit-8 (CCK-8; cat. no. CA1210-100; Beijing Solarbio Science \& Technology Co., Ltd.) were added to each well. The absorbance at $450 \mathrm{~nm}$ was measured after culture for $1 \mathrm{~h}$ without light. The final cell viability of each group was calculated with the following formula: Cell viability $(\%)=($ experimental group-blank $) /($ MIRI only group-blank) x100.

Determination of oxidation index. After ischemia-reperfusion treatment, total proteins were extracted using RIPA lysis buffer (cat. no. R0020; Beijing Solarbio Science \& Technology Co., Ltd.). BCA Protein Assay Kit (cat. no. CW0014; CoWin Biosciences) was used to measure the protein concentration. According to the instructions of the Lipid Peroxidation malondialdehyde (MDA) Assay Kit (cat. no. S0131; Beyotime Institute of Biotechnology), freshly prepared MDA detection solution was added to blank, standard and sample tubes, heated at $100^{\circ} \mathrm{C}$ for $15 \mathrm{~min}$, centrifuged at room temperature and $1,000 \mathrm{x} \mathrm{g}$ for $10 \mathrm{~min}$, and $200 \mu \mathrm{l}$ of each supernatant were placed in a 96 -well plate. The absorbance was determined at $530 \mathrm{~nm}$ using microplate reader (Bio-Rad Laboratories, Inc.) and the absolute value of MDA of each group was calculated. The MDA content per weight of protein $(\mathrm{nmol} / \mathrm{mg}$ ) was calculated according to the protein concentration of each group.

Determination of lactate dehydrogenase ( $L D H)$ in the culture medium. According to the instructions of the LDH Cytotoxicity Assay Kit (cat. no. C0017; Beyotime Institute of Biotechnology), after the ischemia-reperfusion treatment, the culture medium of each group was centrifuged for $5 \mathrm{~min}$ at room temperature and $400 \mathrm{x} \mathrm{g}$, and $120 \mu \mathrm{l}$ supernatant of each sample were placed in a 96-well plate. LDH detection solution $(60 \mu \mathrm{l})$ was added to each well for a 30-min incubation at room temperature. The optical density was measured at $490 \mathrm{~nm}$ using a microplate reader, and the amount of $\mathrm{LDH}(\mathrm{mU} / \mathrm{ml})$ released by the cells in each group was determined. 
mRNA expression of IL-1 $\beta$ and TNF- $\alpha$ in H9c2 cells. The mRNA expression of inflammatory factors in $\mathrm{H} 9 \mathrm{c} 2$ cells was determined via reverse transcription-quantitative PCR (RT-qPCR). Total RNA was extracted using TRIzol $^{\circledR}$ reagent (cat. no. 15596018; Thermo Fisher Scientific, Inc.), and the RNA concentration was quantified in each group. Complementary DNA (cDNA) was obtained from RNA using HiScript II Q RT SuperMix for $\mathrm{qPCR}+\mathrm{gDNA}$ wiper according to the manufacturer's instructions. A PCR Master Mix of $10 \mu \mathrm{l}$ (4.8 $\mu \mathrm{l} \mathrm{cDNA}$ template; $5 \mu 1$ qPCR Master Mix; $0.2 \mu 1$ gene primers) and a LightCycler ${ }^{\circledast} 480$ system II [Roche Diagnostics (Shanghai) Co., Ltd.] were used to perform qPCR. The following thermocycling conditions were used for qPCR: Pre-denaturation at $95^{\circ} \mathrm{C}$ for $5 \mathrm{~min} ; 40$ cycles of $95^{\circ} \mathrm{C}$ for $10 \mathrm{sec}$ and $60^{\circ} \mathrm{C}$ for $30 \mathrm{sec}$. The expression levels were normalized to GAPDH. The primers used were as follows: TNF- $\alpha$ forward, 5'-TGA TCGGTCCCAACAAGGA-3' and reverse, 5'-TGCTTGGTG GTTTGCTACGA-3'; IL-1 $\beta$ forward, 5'-GGGATGATGACG ACCTGC-3' and reverse, 5'-CCACTTGTTGGCTTATGTT-3'; GAPDH forward, 5'-GTTACCAGGGCTGCCTTCTC-3' and reverse, 5'-ACCAGCTTCCCATTCTCAGC-3'. The mRNA expression levels were calculated using the $2^{-\Delta \Delta C q}$ method (29).

Western blot analysis. After establishing the MIRI model, the original medium was discarded and H9c2 cells were washed three times using TBS with Tween-20 (TBST; cat. no. T1085; Beijing Solarbio Science \& Technology Co., Ltd.). A total of $120 \mu \mathrm{l}$ RIPA lysis buffer was added to each $25-\mathrm{cm}^{2}$ culture flask. The lysates were centrifuged at $4^{\circ} \mathrm{C}$ and $12,000 \mathrm{x} \mathrm{g}$ for 5 min. BCA Protein Assay Kit was used to measure protein concentration. The protein samples were mixed with $5 \mathrm{X}$ loading buffer (cat. no. P1015; Beijing Solarbio Science \& Technology Co., Ltd.) at a $4: 1$ ratio, and subsequently boiled at $100^{\circ} \mathrm{C}$ for $5 \mathrm{~min}$. A total of $30 \mu \mathrm{g}$ protein/sample were separated by $10 \%$ SDS-PAGE. The proteins were transferred onto PVDF membranes (MilliporeSigma), which were then blocked with $5 \%$ non-fat milk at room temperature for $2 \mathrm{~h}$. The membranes were incubated at $4^{\circ} \mathrm{C}$ overnight with primary antibodies as follows: Anti-PI3K (1:10,000; cat. no. 09-482; MilliporeSigma); anti-AKT (1:2,000; cat. no. 05-591; MilliporeSigma); anti-phosphorylated (p)-AKT (1:7,000; cat. no. ab81283; Abcam); anti-HSP70 (1:1,000; cat. no. ab181606; Abcam) or anti-GAPDH (1:10,000; cat. no. ab181602; Abcam). After washing with TBST three times, the goat anti-rabbit immunoglobulin $\mathrm{G}(\mathrm{IgG} ; \mathrm{H}+\mathrm{L})$ horseradish peroxidase (HRP; 1:5,000; cat. no. GAR007; MultiSciences Biotech Co., Ltd.) or goat anti-mouse $\operatorname{IgG}(\mathrm{H}+\mathrm{L})$ HRP (1:5,000 dilution, GAM007; MultiSciences Biotech Co., Ltd.) was incubated with the membrane for $2 \mathrm{~h}$ at room temperature, and the membranes were subsequently washed three times with TBST. Protein bands were visualized using ECL detection reagents (cat. no. CW0049M; CWBIO). The relative band intensity was measured by Image-Pro Plus 6.0 software (Media Cybernetics, Inc.).

Immunofluorescence staining. Cells were cultured for 24 to $48 \mathrm{~h}$ before the MIRI model was established. Subsequently, the cells were washed three times with PBS and fixed with 4\% paraformaldehyde (cat. no. P1110; Beijing Solarbio Science \& Technology Co., Ltd.) at room temperature for 30 min, then blocked with $10 \%$ goat serum (cat. no. SL038;
Beijing Solarbio Science \& Technology Co., Ltd.) at room temperature for $30 \mathrm{~min}$. The cells were incubated with the primary antibody overnight at $4^{\circ} \mathrm{C}$ using PI3K (1:5,000; cat. no. 09-482; MilliporeSigma), phosphorylated (p)-Akt (1:200; cat. no. ab81283; Abcam), and HSP70 (1:50; cat. no. ab181606; Abcam). The cells were then washed and incubated with goat anti-mouse IgG-FITC (1:500; cat. no. SA0015; Beijing Solarbio Science \& Technology Co., Ltd.) or goat anti-rabbit IgG-FITC (1:500; cat. no. SA0025; Beijing Solarbio Science \& Technology Co., Ltd.) for $30 \mathrm{~min}$ without light at $37^{\circ} \mathrm{C}$. Nuclei were stained with a DAPI solution (cat. no. C0065; Beijing Solarbio Science \& Technology Co., Ltd.) at $37^{\circ} \mathrm{C}$ for $5 \mathrm{~min}$. The cells were mounted with an anti-fluorescence attenuator and the staining was observed under a fluorescence microscope.

Statistical analysis. SPSS v20.0 statistical software (IBM Corp.) was used to analyze the data. Results are presented as the mean \pm SEM of three experimental repeats. Significant differences were determined using one-way ANOVA followed by Tukey's test. $\mathrm{P}<0.05$ was considered to indicate a statistically significant difference.

\section{Results}

Effect of different concentrations of NBP on the viability of $H 9 c 2$ cells. The viability of $\mathrm{H} 9 \mathrm{c} 2$ cells treated with different NBP doses was examined via the CCK- 8 colorimetric assay. Compared with the MIRI group, $100 \mu \mathrm{M}$ NBP increased cell viability $(\mathrm{P}<0.05$; Fig. 1A). However, NBP concentrations $\geq 200 \mu \mathrm{M}$ slightly decreased cell viability (Fig. 1A). The present results indicated that $100 \mu \mathrm{M}$ NBP was the optimal concentration for protecting cells from MIRI.

NBP inhibits the oxidative stress response during MIRI. When ischemia-reperfusion injury occurs in cardiomyocytes, oxidative stress also plays an important role (30). An MDA kit was used to explore the effect of NBP on the oxidative stress index after MIRI and the role of PI3K in this process. The oxidation level in the MIRI group was significantly higher compared with the CON group $(\mathrm{P}<0.05)$. However, NBP pretreatment significantly decreased oxidative stress $(\mathrm{P}<0.05)$. In contrast, LY294002 eliminated the antioxidant effect of NBP $(\mathrm{P}<0.05$; Fig. 1B). Namely, NBP significantly decreased the oxidative stress response of $\mathrm{H} 9 \mathrm{c} 2$ cells during MIRI, but this antioxidant protective effect was eliminated by blocking PI3K.

NBP protects $H 9 c 2$ cells from MIRI. In vitro, the destruction of the cell membrane structure caused by apoptosis or necrosis leads to LDH release into the culture medium (31). Therefore, LDH activity can indirectly indicate the degree of cell damage. To explore the effect of NBP on MIRI and the involvement of PI3K in MIRI, H9c2 cell viability was determined via CCK-8 colorimetric assay, while an LDH kit was used to determine the LDH content in the culture medium. Compared with the CON group, the cell viability of the MIRI group was significantly lower $(\mathrm{P}<0.05)$. However, the cell viability of the MIRI + NBP group was higher than that of the MIRI group $(\mathrm{P}<0.05)$. By contrast, addition of the PI3K inhibitor LY294002 decreased cell viability $(\mathrm{P}<0.05$; Fig. $1 \mathrm{C})$. Furthermore, the $\mathrm{LDH}$ content 
A

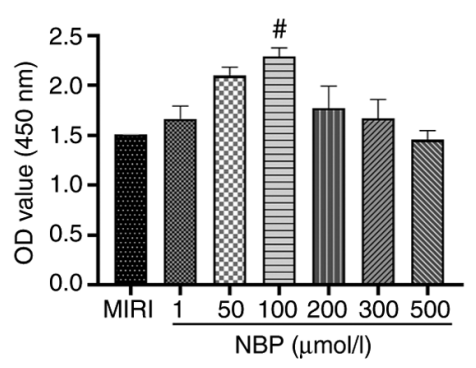

D

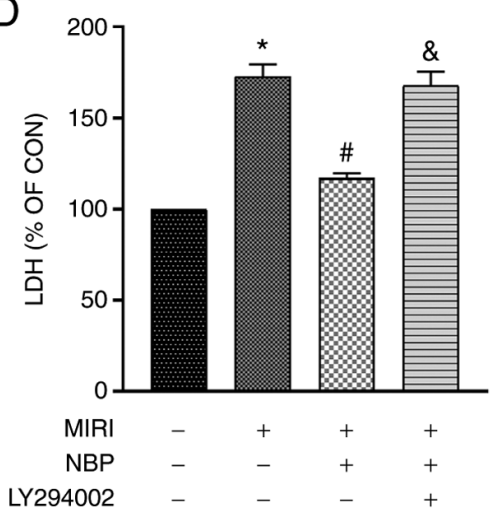

$\mathrm{B}$

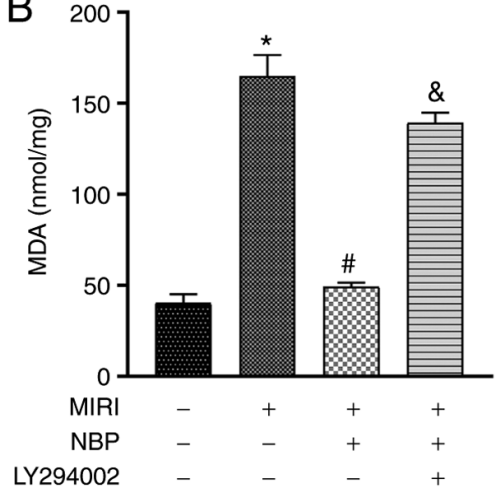

$\mathrm{E}$

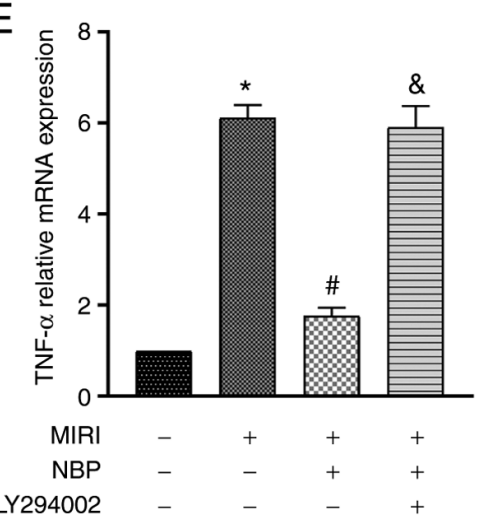

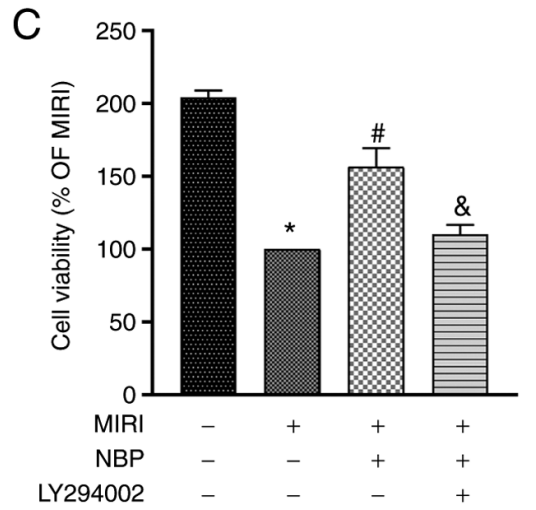

$\mathrm{F}$

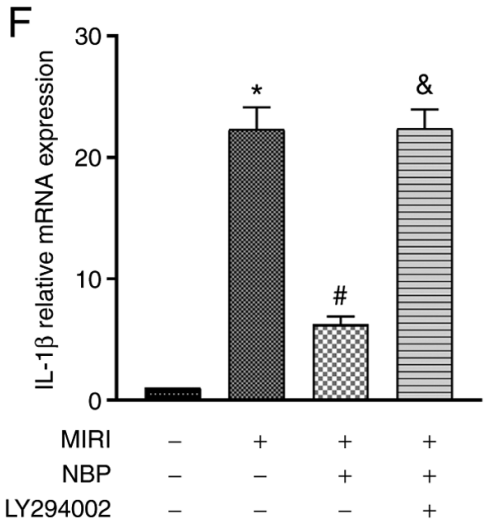

Figure 1. Effects of NBP on H9c2 cell viability, degree of oxidative stress reaction, level of cell injury and mRNA expression of TNF- $\alpha$ and IL-1 $\beta$ in different groups after MIRI. (A) Cell viability after treatment with different NBP concentrations. (B) Lipid peroxidation MDA content was determined via an MDA assay kit. (C) Cell viability percentages. (D) The release of LDH was determined using an LDH release assay kit. mRNA expression of (E) TNF- $\alpha$ and (F) IL-1 $\beta$ was assessed by reverse transcription-quantitative PCR in each group. Results are expressed as the mean \pm SEM. ${ }^{*} \mathrm{P}<0.05$ vs. CON; ${ }^{~} \mathrm{P}<0.05$ vs. MIRI; \&P<0.05 vs. MIRI + NBP. NBP, DL-3-n-butylphthalide; MIRI, myocardial ischemia-reperfusion injury; OD, optical density; MDA, malondialdehyde; LDH, lactate dehydrogenase; CON, control.

of the MIRI group was significantly higher than that of the CON group $(\mathrm{P}<0.05)$. Pretreatment with NBP decreased the LDH release $(\mathrm{P}<0.05)$, but addition of LY294002 reversed this effect $(\mathrm{P}<0.05$; Fig. 1D). Thus, NBP significantly increased the viability of $\mathrm{H} 9 \mathrm{c} 2$ cells after ischemia-reperfusion injury and reduced $\mathrm{H} 9 \mathrm{c} 2$ cell injury. However, the PI3K inhibitor LY294002 reversed the protective effect of NBP on H9c2 cells.

$N B P$ inhibits inflammation by decreasing the TNF- $\alpha$ and IL-1 $\beta$ $m R N A$ expression. Activation of inflammatory cytokines during MIRI aggravates the injury of cardiomyocytes (32). Therefore, to determine the association between the protective effect of NBP during MIRI and inflammatory factors, the mRNA expression of TNF- $\alpha$ and IL-1 $\beta$ was examined in H9c 2 cells via $\mathrm{RT}$-qPCR. Compared with the CON group, the expression of TNF- $\alpha$ and IL-1 $\beta$ in the MIRI group significantly increased $(\mathrm{P}<0.05)$. However, the expression of these genes decreased in the MIRI + NBP compared with the MIRI group $(\mathrm{P}<0.05)$. By contrast, addition of LY294002 increased the expression of TNF- $\alpha$ and IL- $1 \beta$ compared with the MIRI + NBP group $(\mathrm{P}<0.05$; Fig. $1 \mathrm{E}$ and $\mathrm{F})$. Therefore, it was revealed that NBP effectively reduced the expression of inflammatory factors during MIRI, while the application of the PI3K inhibitor LY294002 reversed this anti-inflammatory effect.

NBP activates the HSP70 and PI3K/AKT signaling pathway. During MIRI, the expression of the stress-protective proteins
HSP70 and PI3K/AKT was upregulated. Western blotting and immunofluorescence staining were used to determine the protein expression of HSP70 and PI3K/AKT after NBP treatment. The expression levels of p-AKT and PI3K in the MIRI group were significantly increased compared with the CON group $(\mathrm{P}<0.05)$. In the MIRI + NBP group, the expression of p-AKT and PI3K was increased compared with the MIRI group $(\mathrm{P}<0.05)$. By contrast, in the MIRI + NBP + LY294002 group, the expression of $\mathrm{p}-\mathrm{AKT}$ and $\mathrm{PI} 3 \mathrm{~K}$ decreased compared with the MIRI + NBP group $(\mathrm{P}<0.05$; Fig. 2A-D). This tendency was also observed in the immunofluorescence assay (Fig. 2E and F). Additionally, the expression level of HSP70 in the MIRI group increased compared with the CON group $(\mathrm{P}<0.05)$. NBP pretreatment further increased the expression of HSP70 (P<0.05), whereas LY294002 decreased it compared with the MIRI + NBP group $(\mathrm{P}<0.05$; Fig. $3 \mathrm{~A}$ and $\mathrm{B})$. This tendency was also observed in the immunofluorescence assay (Fig. 3C). The present results indicated that NBP pretreatment could increase the expression level of PI3K, p-AKT and HSP70 following MIRI, and that the inhibition of the PI3K pathway also altered HSP70 expression.

\section{Discussion}

MIRI mechanism involves several processes, including energy metabolism disorder, oxidative stress, calcium overload, inflammation, apoptosis and autophagy (33). In the clinic, the 


\section{A}
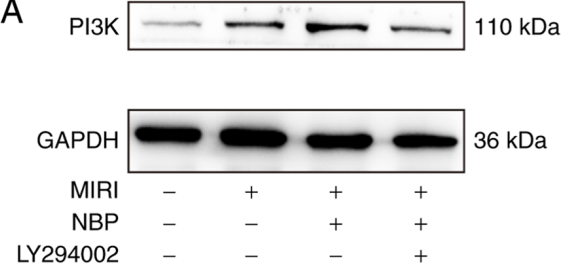

C

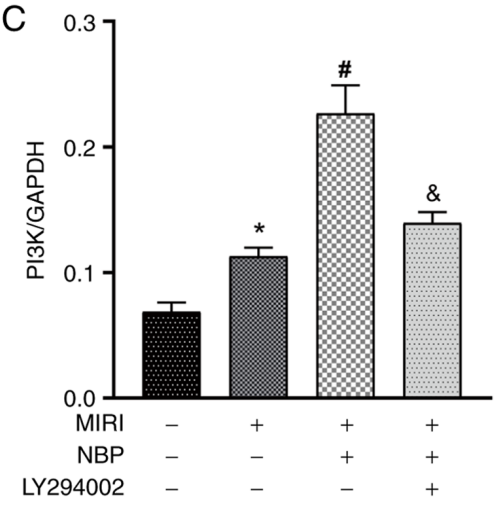

$\mathrm{E}$
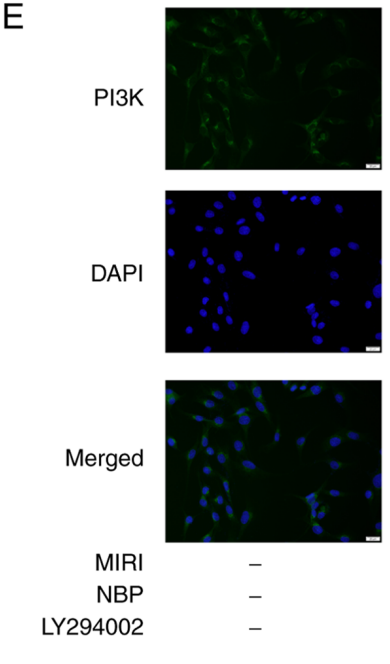

F

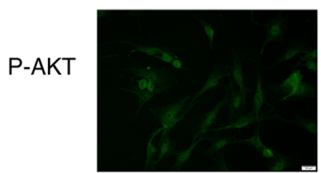

DAPI
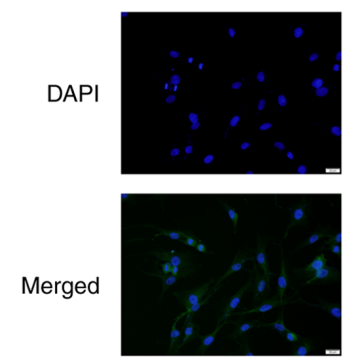

MIRI

NBP

LY294002
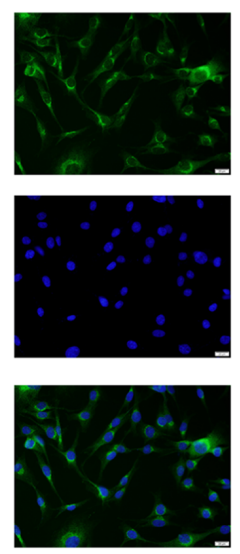

$+$
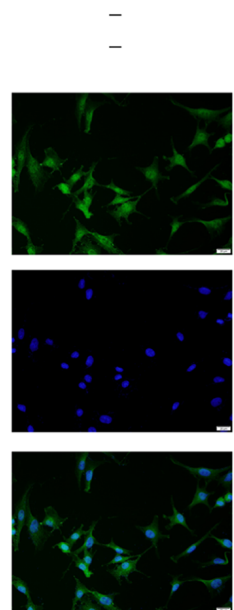

$+$
B

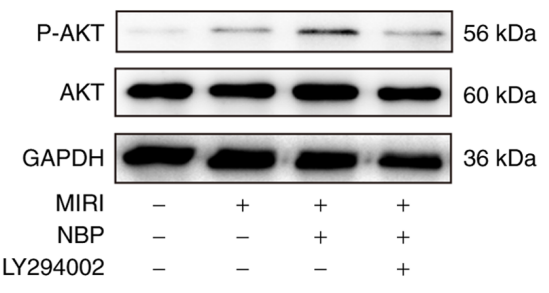

D
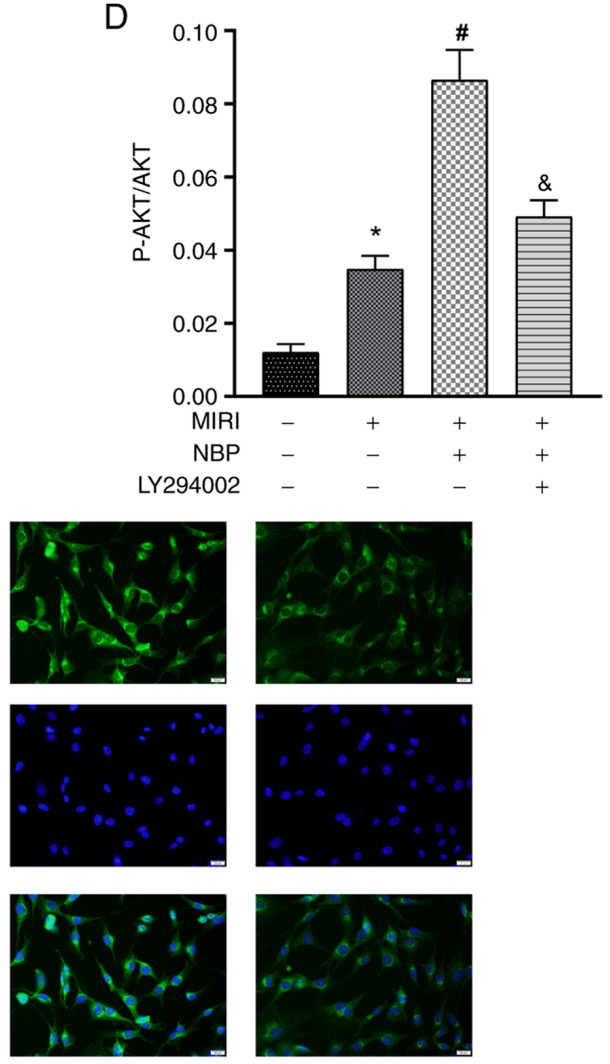

$+$
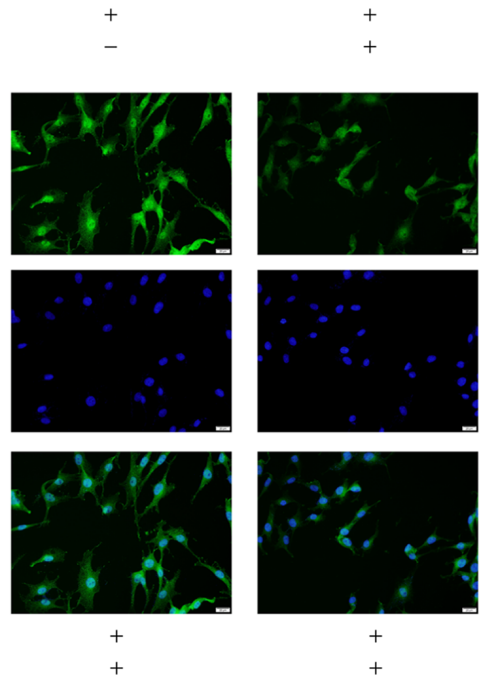

Figure 2. Protein expression of PI3K, AKT and p-AKT in H9c2 cells in different groups after MIRI using western blotting and immunofluorescence. (A) PI3K and (B) AKT and p-AKT expression in H9c2 cells of each group assessed via western blotting. Quantification of the relative expression of (C) PI3K and (D) p-AKT. Results are expressed as the mean \pm SEM. ${ }^{*} \mathrm{P}<0.05$ vs. control; ${ }^{*} \mathrm{P}<0.05$ vs. MIRI; ${ }^{\&} \mathrm{P}<0.05$ vs. MIRI $+\mathrm{NBP}$. Immunofluorescence staining demonstrating (E) PI3K and (F) p-AKT expression in H9c2 cells of each group. Green represents PI3K or p-AKT and blue represents the nuclei. Scale bar, $20 \mu \mathrm{m}$. NBP, DL-3-n-butylphthalide; MIRI, myocardial ischemia-reperfusion injury; p, phosphorylated.

best treatment for patients with myocardial infarction is immediate reperfusion, causing a paradoxical situation because of its adverse effects (34). Therefore, an in-depth understanding of the mechanism of MIRI has become a priority. 
A

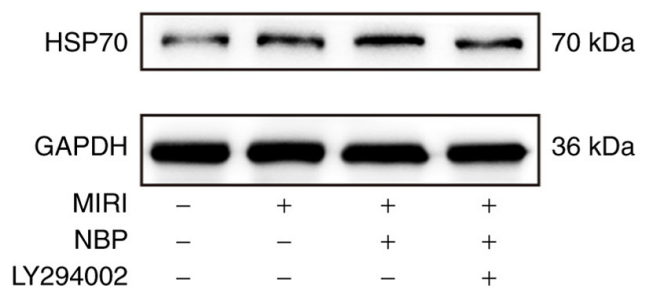

C HSP70
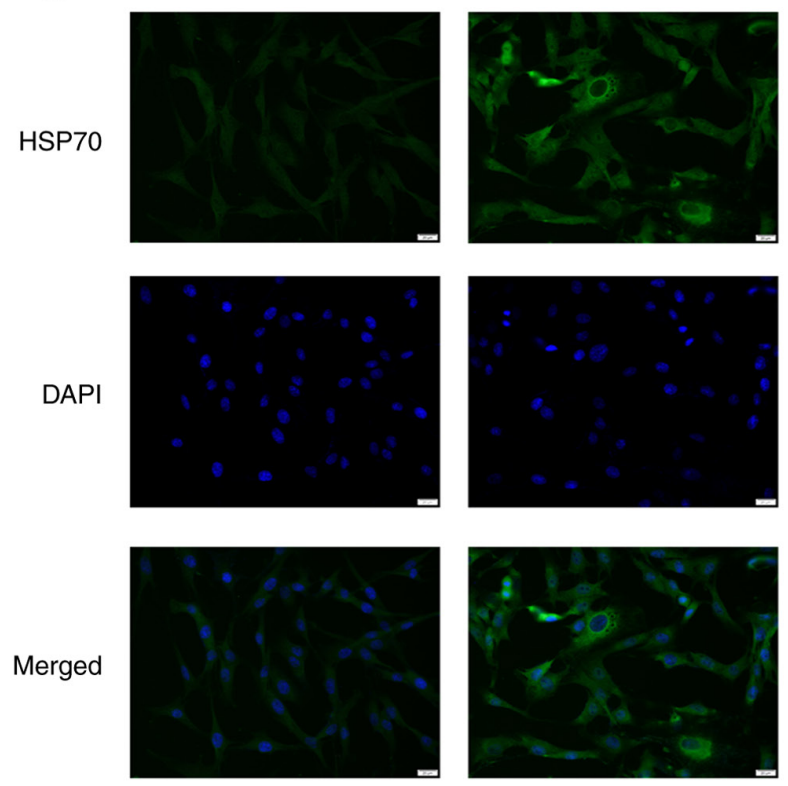

MIRI

NBP

LY294002

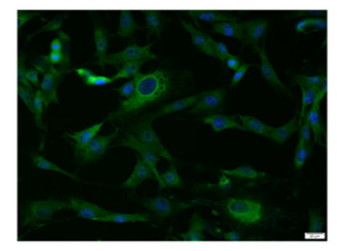

$+$

$-$
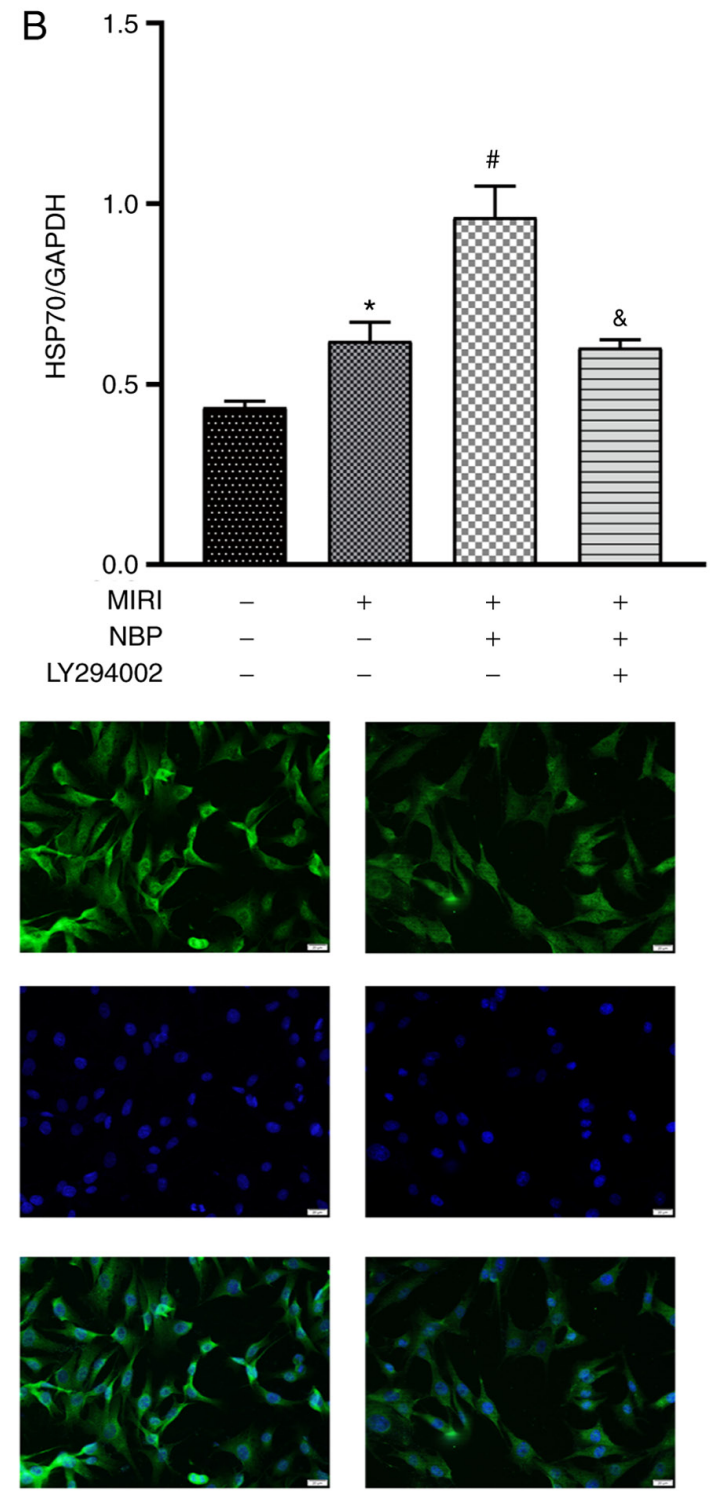

$+$

$+$

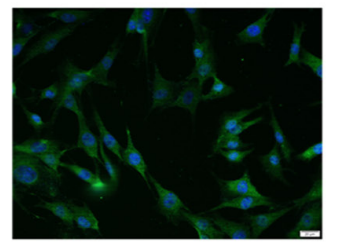

$+$

$+$

Figure 3. Protein expression of HSP70 in H9c2 cells in different groups after MIRI using western blotting and immunofluorescence. (A) HSP70 expression in $\mathrm{H} 9 \mathrm{c} 2$ cells of each group and (B) quantification of the relative expression. Results are expressed as the mean $\pm \mathrm{SEM}$. ${ }^{*} \mathrm{P}<0.05 \mathrm{vs}$. control; ${ }^{*} \mathrm{P}<0.05$ vs. MIRI; ${ }^{\&} \mathrm{P}<0.05$ vs. MIRI + NBP. (C) Immunofluorescence staining demonstrating HSP70 expression in H9c2 cells of each group. Green represents HSP70 and blue represents the nuclei. Scale bar=20 $\mu \mathrm{m}$. NBP, DL-3-n-butylphthalide; MIRI, myocardial ischemia-reperfusion injury; HSP70, heat shock protein 70.

As a drug used for the treatment of stroke, NBP has been widely studied in the nervous system. For instance, a previous study has indicated that NBP could reduce nerve cell death during cerebral ischemia by inhibiting inflammation, oxidative stress, autophagy and apoptosis (35). NBP has a wide range of functions, as it can protect the nervous system and delay the onset and progression of diabetic cataract $(25,36)$. Furthermore, a previous study has revealed that NBP exhibited a protective effect against MIRI, and its mechanism may be associated with the regulation of the mitochondrial apoptosis and the AKT/Nuclear factor erythroid 2-related factor 2 signaling pathways (37). However, the specific mechanism underlying the NBP effect on MIRI needs to be further explored.

As a protective protein under stress, HSP70 has been indicated to play a protective role in cardiomyocytes during
MIRI (38). However, it is unclear whether HSP70 is involved in the NBP protective effect on cardiomyocytes. The present results indicated that NBP regulated the expression of HSP70 via the PI3K/AKT signaling pathway, thereby protecting H9c2 cells from MIRI. The present study provided a basis for the application of NBP in the clinical treatment of cardiovascular diseases, and elucidated the protective mechanism of NBP to a certain extent.

The current study determined the optimal concentration of NBP pretreatment against MIRI by establishing a concentration gradient of NBP, indicating that $100 \mu \mathrm{M}$ NBP exhibited the strongest protective effect compared with the other concentrations. NBP pretreatment improved the decrease in cell viability induced by MIRI and significantly reduced LDH release, thus protecting H9c2 cells from MIRI. LY294002 treatment 
reversed the protective effect of NBP, decreased cell viability and increased $\mathrm{LDH}$ release. The present results suggested that the protective effect of NBP on $\mathrm{H} 9 \mathrm{c} 2$ cells depended on the activation of the PI3K signaling pathway.

During myocardial ischemia, a large number of oxygen free radicals are produced, eventually leading to the destruction of the mitochondrial structure, mitochondrial swelling, cell membrane structure damage and the disturbance of cell energy metabolism (39). MDA is the final product of reactive oxygen species oxidation of arachidonic acid, representing a biomarker of lipid peroxidation produced by oxidative stress (40). The present results indicated that the MDA content in H9c2 cells significantly increased after MIRI; however, the oxidation index indicated by the MDA content significantly decreased after NBP pretreatment. By contrast, the oxidation index increased again after LY294002 intervention. NBP inhibited oxidative stress in a process of myocardial protection, and this antioxidant mechanism was blocked by inhibiting the PI3K pathway.

A number of inflammatory cytokines are activated during MIRI, which can cause systemic inflammation (41,42). TNF- $\alpha$ causes local inflammation and apoptosis, eventually contributing to cardiac insufficiency and even cardiac infarction (43). IL-1 plays a central role in human autoinflammatory diseases and its target genes are numerous, such as IL-1 $\beta$, IL-6 and IL-8 (44). Among those, IL-1 $\beta$ expression was quantified in the present study. IL-1 $\beta$ is a typical pro-inflammatory factor that mediates the infiltration of neutrophils and macrophages in local tissues during ischemia-reperfusion, which causes myocardial fibrosis and structural remodeling (45). To elucidate the effect of NBP on H9c2 cells, RT-qPCR was performed to determine the expression level of inflammatory cytokines. The present results indicated that the mRNA expression of IL-1 $\beta$ and TNF- $\alpha$ in the MIRI group significantly increased compared with the CON group. Pretreatment with NBP significantly inhibited the inflammatory response; however, this anti-inflammatory effect was reversed by blocking the PI3K signaling pathway. Namely, blocking the PI3K pathway eliminated the inflammatory inhibitory effect of NBP.

The protective role of the PI3K/AKT signaling pathway against MIRI is well-known. For instance, a previous study has demonstrated that activation of the insulin-induced PI3K/AKT pathway could inhibit cardiomyocyte apoptosis and protect or improve local and global cardiac function (46). Upon PI3K-induced activation, AKT activates GSK-3 $\beta$ through phosphorylation, this junction of several pathways plays a notable role in myocardial protection (47). Relevant experiments have confirmed that the increase of heat shock factor 1 (HSF1) results from GSK-3 $\beta$ phosphorylation $(48,49)$. HSP70 is a stress-protective protein that protects cardiomyocytes during MIRI (50). HSP70 induction is mediated by the interaction with HSF1; therefore, PI3K/AKT and HSP70 may be associated through GSK-3 $\beta$ and $\operatorname{HSF} 1(51,52)$. It was previously demonstrated that there is a mutual regulation between the PI3K/AKT pathway and HSP70 in brain and lung tissues $(16,17)$. To further elucidate the association between the PI3K/AKT pathway and HSP70 in H9c2 cells, the expression of HSP70 was examined upon application of the PI3K inhibitor LY294002. Western blotting and immunofluorescence staining indicated that MIRI increased the expression levels of HSP70, PI3K and p-AKT, and that NBP pretreatment further increased this expression. By contrast, LY294002 suppressed the expression levels of PI3K, p-AKT and HSP70. Therefore, the protective effect of NBP against MIRI was indicated to be associated with the PI3K/AKT signaling pathway and HSP70.

Certain main limitations can be taken into account in the present work. Firstly, the present regulation pathway needs to be further investigated in an animal model. Secondly, the protective effect of NBP on MIRI has not been reflected in clinical treatment, and further studies are required to explore the drug administration route and dosages. Thirdly, H9c2 cells are rat myoblasts and not cardiomyocytes; therefore, they present differences in their characteristics and protein expression compared with adult cardiomyocytes. Future studies will investigate the present hypotheses in primary cardiomyocytes.

In summary, NBP upregulated HSP70 through the $\mathrm{PI} 3 \mathrm{~K} / \mathrm{AKT}$ pathway and reduced the inflammatory response, oxidative stress and injury of H9c2 cells, thereby attenuating MIRI. These findings may provide a novel therapeutic target for the clinical treatment of MIRI.

\section{Acknowledgements}

Not applicable.

\section{Funding}

The present work was supported by National Natural Science Foundation of China (grant no. 81870593) and Natural Science Foundation of Shandong Province of China (grant nos. ZR201 8MH008 and ZR2019PH037).

\section{Availability of data and materials}

The datasets used and/or analyzed during the current study are available from the corresponding author on reasonable request.

\section{Authors' contributions}

WH, XDS and XTS designed the research and revised the manuscript. YY, YZ and YL conducted experiments. MW and $\mathrm{BD}$ analyzed the data and participated in technical editing of the manuscript. YY and XTS wrote the draft manuscript. YY and WH confirm the authenticity of all the raw data. All authors read and approved the final manuscript.

\section{Ethics approval and consent to participate}

Not applicable.

\section{Patient consent for publication}

Not applicable.

\section{Competing interests}

The authors declare that they have no competing interests. 


\section{References}

1. Wang BF and Yoshioka J: The emerging role of thioredoxin-interacting protein in myocardial ischemia/reperfusion injury. J Cardiovasc Pharmacol Ther 22: 219-229, 2017.

2. Li X, Liu M, Sun R, Zeng Y, Chen S and Zhang P: Protective approaches against myocardial ischemia reperfusion injury. Exp Ther Med 12: 3823-3829, 2016.

3. Chin KY, Qin C, May L, Ritchie RH and Woodman OL: New pharmacological approaches to the prevention of myocardial ischemia-reperfusion injury. Curr Drug Targets 18: 1689-1711, 2017.

4. Esposito ML, Zhang Y, Qiao X, Reyelt L, Paruchuri V, Schnitzler GR, Morine KJ, Annamalai SK, Bogins C, Natov PS, et al: Left ventricular unloading before reperfusion promotes functional recovery after acute myocardial infarction. J Am Coll Cardiol 72: 501-514, 2018.

5. Wang Z, Wang Y, Ye J, Lu X, Cheng Y, Xiang L, Chen L, Feng W, Shi H, Yu X, et al: bFGF attenuates endoplasmic reticulum stress and mitochondrial injury on myocardial ischaemia/reperfusion via activation of PI3K/Akt/ERK1/2 pathway. J Cell Mol Med 19: 595-607, 2015.

6. Radons J: The human HSP70 family of chaperones: Where do we stand? Cell Stress Chaperones 21: 379-404, 2016.

7. Guo S, Gao C, Xiao W, Zhang J, Qu Y, Li J and Ye F: Matrine protects cardiomyocytes from ischemia/reperfusion injury by regulating HSP70 expression via activation of the JAK2/STAT3 pathway. Shock 50: 664-670, 2018.

8. Wright MA, Aprile FA, Bellaiche MM, Michaels TC, Muller T, Arosio P, Vendruscolo M, Dobson CM and Knowles TP: Cooperative assembly of Hsp70 subdomain clusters. Biochemistry 57: 3641-3649, 2018.

9. Tang L, Mo Y, Li Y, Zhong Y, He S, Zhang Y, Tang Y, Fu S, Wang $X$ and Chen A: Urolithin A alleviates myocardial ischemia/reperfusion injury via PI3K/Akt pathway. Biochem Biophys Res Commun 486: 774-780, 2017.

10. Zhang BF, Jiang H, Chen J, Guo X, Li Y, Hu Q and Yang S: Nobiletin ameliorates myocardial ischemia and reperfusion injury by attenuating endoplasmic reticulum stress-associated apoptosis through regulation of the PI3K/AKT signal pathway. Int Immunopharmacol 73: 98-107, 2019.

11. Thokala S, Inapurapu S, Bodiga VL, Vemuri PK and Bodiga S: Loss of ErbB2-PI3K/Akt signaling prevents zinc pyrithione-induced cardioprotection during ischemia/reperfusion. Biomed Pharmacother 88: 309-324, 2017.

12. Vanhaesebroeck B, Guillermet-Guibert J, Graupera M and Bilanges B: The emerging mechanisms of isoform-specific PI3K signalling. Nat Rev Mol Cell Biol 11: 329-341, 2010.

13. Sulaiman D, Li J, Devarajan A, Cunningham CM, Li M, Fishbein GA, Fogelman AM, Eghbali M and Reddy ST: Paraoxonase 2 protects against acute myocardial ischemia-reperfusion injury by modulating mitochondrial function and oxidative stress via the PI3K/Akt/GSK-3 $\beta$ RISK pathway. J Mol Cell Cardiol 129: 154-164, 2019.

14. Hausenloy DJ and Yellon DM: New directions for protecting the heart against ischaemia-reperfusion injury: Targeting the reperfusion injury salvage kinase (RISK)-pathway. Cardiovasc Res 61: 448-460, 2004.

15. Damilano F, Perino A and Hirsch E: PI3K kinase and scaffold functions in heart. Ann N Y Acad Sci 1188: 39-45, 2010.

16. Zhai C, Lv J, Wang K, Li Q and Qu Y: HSP70 silencing aggravates apoptosis induced by hypoxia/reoxygenation in vitro. Exp Ther Med 18: 1013-1020, 2019.

17. Ji K, Xue L, Cheng J and Bai Y: Preconditioning of H2S inhalation protects against cerebral ischemia/reperfusion injury by induction of HSP70 through PI3K/Akt/Nrf2 pathway. Brain Res Bull 121: 68-74, 2016.

18. Abdoulaye IA and Guo YJ: A review of recent advances in neuroprotective potential of 3-N-Butylphthalide and its derivatives. Biomed Res Int 2016: 5012341, 2016.

19. Zhao Y, Lee JH, Chen D, Gu X, Caslin A, Li J, Yu SP and Wei L: DL-3-n-butylphthalide induced neuroprotection, regenerative repair, functional recovery and psychological benefits following traumatic brain injury in mice. Neurochem Int 111: 82-92, 2017.

20. Qin C, Zhou P, Wang L, Mamtilahun M, Li W, Zhang Z, Yang GY and Wang Y: Dl-3-N-butylphthalide attenuates ischemic reperfusion injury by improving the function of cerebral artery and circulation. J Cereb Blood Flow Metab 39: 2011-2021, 2019.

21. Kawasaki K, Yano K, Sasaki K, Tawara S, Ikegaki I, Satoh S, Ohtsuka Y, Yoshino Y, Kuriyama H, Asano T and Seto M: Correspondence between neurological deficit, cerebral infarct size, and rho-kinase activity in a rat cerebral thrombosis model. J Mol Neurosci 39: 59-68, 2009.
22. Zhao Q,Zhang C, Wang X, Chen L,JiH and Zhang Y: (S)-ZJM-289, a nitric oxide-releasing derivative of 3-n-butylphthalide, protects against ischemic neuronal injury by attenuating mitochondrial dysfunction and associated cell death. Neurochem Int 60: 134-144, 2012.

23. Zhang P, Guo ZF, Xu YM, Li YS and Song JG: N-Butylphthalide (NBP) ameliorated cerebral ischemia reperfusion-induced brain injury via HGF-regulated TLR4/NF- $\mathrm{B}$ signaling pathway. Biomed Pharmacother 83: 658-666, 2016.

24. Liao D, Xiang D, Dang R, Xu P, Wang J, Han W, Fu Y, Yao D, Cao L and Jiang P: Neuroprotective effects of dl-3-n-Butylphthalide against doxorubicin-induced neuroinflammation, oxidative stress, endoplasmic reticulum stress, and behavioral changes. Oxid Med Cell Longev 2018: 9125601, 2018.

25. Wang YG, Li Y, Wang CY, Ai JW, Dong XY, Huang HY, Feng ZY, Pan YM, Lin Y, Wang BX and Yao LL: L-3-n-Butylphthalide protects rats' cardiomyocytes from ischaemia/reperfusion-induced apoptosis by affecting the mitochondrial apoptosis pathway. Acta Physiol (Oxf) 210: 524-533, 2014.

26. Qiu H, Wu H, Ma J, Cao H, Huang L, Qiu W, Peng Y and Ding C: DL-3-n-Butylphthalide reduces atrial fibrillation susceptibility by inhibiting atrial structural remodeling in rats with heart failure. Naunyn Schmiedebergs Arch Pharmacol 391: 323-334, 2018.

27. Qiu H, Ma J, Wu H and Ding C: DL-3-n-butylphthalide improves ventricular function, and prevents ventricular remodeling and arrhythmias in post-MI rats. Naunyn Schmiedebergs Arch Pharmacol 391: 627-637, 2018.

28. Yu P, Ma S, Dai X and Cao F: Elabela alleviates myocardial ischemia reperfusion-induced apoptosis, fibrosis and mitochondrial dysfunction through PI3K/AKT signaling. Am J Transl Res 12: 4467-4477, 2020.

29. Livak KJ and Schmittgen TD: Analysis of relative gene expression data using real-time quantitative PCR and the 2(-Delta Delta C(T)) Method. Methods 25: 402-408, 2001.

30. Zhao D, Yang J and Yang L: Insights for oxidative stress and mTOR signaling in myocardial ischemia/reperfusion injury under diabetes. Oxid Med Cell Longev 2017: 6437467, 2017.

31. Amani M, Jeddi S, Ahmadiasl N, Usefzade N and Zaman J: Effect of HEMADO on level of CK-MB and LDH enzymes after ischemia/reperfusion injury in isolated rat heart. Bioimpacts 3: 101-104, 2013.

32. Xu Z, Wang D, Zhou Z, Chen Q, Zhang D, Chen S, Jiang H, Jia C and Liu X: Dexmedetomidine attenuates renal and myocardial ischemia/reperfusion injury in a dose-dependent manner by inhibiting inflammatory response. Ann Clin Lab Sci 49: 31-35, 2019.

33. Zhang C, He M, Ni L, He K, Su K, Deng Y, Li Y and Xia H: The role of arachidonic acid metabolism in myocardial ischemia-reperfusion injury. Cell Biochem Biophys 78: 255-265, 2020.

34. Liu H, Yang L, Wu HJ, Chen KH, Lin F, Li G, Sun HY, Xiao GS, Wang Y and Li GR: Water-soluble acacetin prodrug confers significant cardioprotection against ischemia/reperfusion injury. Sci Rep 6: 36435, 2016.

35. Wang S, Ma F, Huang L, Zhang Y, Peng Y, Xing C, Feng Y, Wang X and Peng Y: Dl-3-n-Butylphthalide (NBP): A promising therapeutic agent for ischemic stroke. CNS Neurol Disord Drug Targets 17: 338-347, 2018.

36. Wang F, Ma J, Han F, Guo X, Meng L, Sun Y, Jin C, Duan H, Li $\mathrm{H}$ and Peng Y: DL-3-n-butylphthalide delays the onset and progression of diabetic cataract by inhibiting oxidative stress in rat diabetic model. Sci Rep 6: 19396, 2016.

37. Bai M, Pan CL, Jiang GX, Zhang YM and Zhang Z: Effects of butylphthalide on oxidative stress and inflammatory response in rats with myocardial infarction through Akt/Nrf2 signaling pathway. Eur Rev Med Pharmacol Sci 23: 9642-9650, 2019.

38. Liu X, Zhang C, Zhang C, Li J, Guo W, Yan D, Yang C, Zhao J, Xia T, Wang Y, et al: Heat shock protein 70 inhibits cardiomyocyte necroptosis through repressing autophagy in myocardial ischemia/reperfusion injury. In Vitro Cell Dev Biol Anim 52: 690-698, 2016.

39. Yuan X, Juan Z, Zhang R, Sun X, Yan R, Yue F, Huang Y, Yu J and $\mathrm{Xia} \mathrm{X}$ : Clemastine fumarate protects against myocardial ischemia reperfusion injury by activating the TLR4/PI3K/Akt signaling pathway. Front Pharmacol 11: 28, 2020.

40. Tsikas D: Assessment of lipid peroxidation by measuring malondialdehyde (MDA) and relatives in biological samples: Analytical and biological challenges. Anal Biochem 524: 13-30, 2017. 
41. Ma C, Xu Z and Lv H: Low n-6/n-3 PUFA ratio improves inflammation and myocardial ischemic reperfusion injury. Biochem Cell Biol 97: 621-629, 2019.

42. Xiong J, Yuan YJ, Xue FS, Wang Q, Cheng Y, Li RP, Liao X and Liu JH: Postconditioning with $\alpha 7 \mathrm{nAChR}$ agonist attenuates systemic inflammatory response to myocardial ischemia-reperfusion injury in rats. Inflammation 35: 1357-1364, 2012.

43. Sun M, Dawood F, Wen WH, Chen M, Dixon I, Kirshenbaum LA and Liu PP: Excessive tumor necrosis factor activation after infarction contributes to susceptibility of myocardial rupture and left ventricular dysfunction. Circulation 110: 3221-3228, 2004.

44. Weber A, Wasiliew P and Kracht M: Interleukin-1 (IL-1) pathway. Sci Signal 3: cm1, 2010

45. Neri M, Fineschi V, Di Paolo M, Pomara C, Riezzo I, Turillazzi E and Cerretani D: Cardiac oxidative stress and inflammatory cytokines response after myocardial infarction. Curr Vasc Pharmacol 13: 26-36, 2015.

46. Yao $H$, Han $X$ and Han $X$ : The cardioprotection of the insulin-mediated PI3K/Akt/mTOR signaling pathway. Am J Cardiovasc Drugs 14: 433-442, 2014.

47. Wei D, Xu H, Gai X and Jiang Y: Astragaloside IV alleviates myocardial ischemia-reperfusion injury in rats through regulating PI3K/AKT/GSK-3 $\beta$ signaling pathways. Acta Cir Bras 34: e201900708, 2019.

48. Juhaszova M, Zorov DB, Yaniv Y, Nuss HB, Wang S and Sollott SJ: Role of glycogen synthase kinase-3beta in cardioprotection. Circ Res 104: 1240-1252, 2009.
49. Son TW, Yun SP, Yong MS, Seo BN, Ryu JM, Youn HY, Oh YM and Han HJ: Netrin-1 protects hypoxia-induced mitochondrial apoptosis through HSP27 expression via DCC- and integrin a6b4-dependent Akt, GSK-3 $\beta$, and HSF-1 in mesenchymal stem cells. Cell Death Dis 4: e563, 2013.

50. Nair SP and Sharma RK: Heat shock proteins and their expression in primary murine cardiac cell populations during ischemia and reperfusion. Mol Cell Biochem 464: 21-26, 2020.

51. Xu NW, Chen Y, Liu W, Chen YJ, Fan ZM, Liu M and Li LJ: Inhibition of JAK2/STAT3 signaling pathway suppresses proliferation of burkitt's lymphoma raji cells via cell cycle progression, apoptosis, and oxidative stress by modulating HSP70. Med Sci Monit 24: 6255-6263, 2018.

52. Staib JL, Quindry JC, French JP, Criswell DS and Powers SK: Increased temperature, not cardiac load, activates heat shock transcription factor 1 and heat shock protein 72 expression in the heart. Am J Physiol Regul Integr Comp Physiol 292: R432-R439, 2007.

This work is licensed under a Creative Commons Attribution-NonCommercial-NoDerivatives 4.0 International (CC BY-NC-ND 4.0) License. 